\title{
Spectrophotometric variegation of the layering in comet 67P/Churyumov-Gerasimenko as seen by OSIRIS
}

\author{
G. Tognon ${ }^{1}$, S. Ferrari ${ }^{1}$, L. Penasa ${ }^{1}$, F. La Forgia ${ }^{2}$, M. Massironi ${ }^{1,3}$, G. Naletto ${ }^{1,4,5}$, M. Lazzarin ${ }^{2}$, P. Cambianica ${ }^{1}$, \\ A. Lucchetti ${ }^{6}$, M. Pajola ${ }^{6}$, F. Ferri ${ }^{1}$, C. Güttler ${ }^{7}$, B. Davidsson ${ }^{8}$, P. Deshapriya ${ }^{9}$, S. Fornasier ${ }^{9}$, S. Mottola ${ }^{10}$, I. Tóth $^{11}$, \\ H. Sierks ${ }^{7}$, P. L. Lamy $^{12}$, R. Rodrigo ${ }^{13,14}$, D. Koschny ${ }^{15}$, C. Barbieri ${ }^{2}$, M. A. Barucci ${ }^{9}$, J.-L. Bertaux ${ }^{16}$, I. Bertini ${ }^{2}$, \\ D. Bodewits ${ }^{17}$, G. Cremonese ${ }^{6}$, V. Da Deppo ${ }^{5}$, S. Debei ${ }^{18}$, M. De Cecco ${ }^{19}$, J. Deller ${ }^{7}$, M. Franceschi ${ }^{3}$, E. Frattin ${ }^{2,6}$, \\ M. Fulle ${ }^{20}$, P. J. Gutiérrez ${ }^{7}$, W.-H. Ip ${ }^{21,22}$, H. U. Keller ${ }^{10,23}$, L. M. Lara ${ }^{24}$, J. J. López-Moreno ${ }^{24}$, F. Marzari ${ }^{4}$,
} V. Petropoulou ${ }^{1}, \mathrm{X} . \mathrm{Shi}^{7}$, and C. Tubiana ${ }^{7}$

(Affiliations can be found after the references)

Received 14 December 2018 / Accepted 15 March 2019

\begin{abstract}
Context. Between August 2014 and September 2016, the ESA space mission Rosetta escorted comet 67P/Churyumov-Gerasimenko (67P) during its perihelion passage. The onboard OSIRIS Narrow Angle Camera (NAC) acquired high-resolution multispectral images of the cometary surface. These datasets allowed a characterization of the spectrophotometric variegation of the layering of the large lobe, correlated with the layer structural elevation.

Aims. We perform a spectrophotometric characterization of the outcropping stratification of the small lobe of 67P as a function of its structural elevation, and consequently, a spectrophotometric comparison of the layered outcrops of the two lobes.

Methods. We selected two sequences of post-perihelion OSIRIS NAC images ( 2.4 au outbound to the Sun), from which we built up two multispectral images, framing an extended geological section of the onion-like stratification of the small lobe. Then we classified the consolidated areas of the outcropping and the relative coarse deposits that were identified in the multispectral data with a two-class maximum likelihood method. For this, we defined the classes as a function of the structural elevation of the surface.

Results. As a result, we identified a brightness variegation of the surface reflectance that is correlated with the structural elevation. The outer class, which is located at higher elevations, appears darker than the inner class. This fits previously obtained results for the large lobe. The reflectance values of the nucleus of 67P tend to decrease with increasing structural elevation.

Conclusions. The observed spectrophotometric variegation can be due to a different texture as well as to a different content of volatiles and refractories. We suggest that the outer outcrops appear darker because they have been exposed longer, and the inner outcrops appear brighter because the surface has been more effectively rejuvenated. We interpret this variegation as the result of an evolutionary process.
\end{abstract}

Key words. comets: general - comets: individual: 67P/Churyumov-Gerasimenko - methods: data analysis

\section{Introduction}

Before its controlled landing on the surface of comet 67P/Churyumov-Gerasimenko (hereafter 67P) on September 2016, the ESA Rosetta spacecraft escorted the comet during its approach to the Sun. The images acquired by the Optical, Spectroscopic and Infrared Remote Imaging System (OSIRIS, Keller et al. 2007) allowed describing a cometary nucleus with a bilobed shape (Sierks et al. 2015), with a recognizable large lobe called "body" and a small lobe called "head". They are connected by a narrow region called "neck". The nucleus of 67P is characterized by several geomorphological features such as cliffs, hogbacks, cuestas, mesas, and sets of terraces, and by recurring packs of layers. These layers support the assumption that the nucleus is stratified like an onion. This onion-like stratification independently envelopes both lobes and formed before they merged through a low-velocity collision (Massironi et al. 2015; Davidsson et al. 2016; Penasa et al. 2017).

The OSIRIS observations, and in particular those made by the Narrow Angle Camera (NAC), also permitted deriving the spectrophotometric properties of the cometary body of 67P and thereby to study the heterogeneities of the nucleus in terms of albedo, texture, and composition. Fornasier et al. (2015) found that in the $250-1000 \mathrm{~nm}$ wavelength range, the nucleus shows a red-sloped spectrum with no clear absorption bands, similarly to other cometary nuclei. The nucleus of $67 \mathrm{P}$ has a dark surface in absolute terms overall, with an estimated geometric albedo of $6.5 \pm 0.2 \%$ at $649 \mathrm{~nm}$. Albedo and spectral slopes, computed in the 535-882 $\mathrm{nm}$ range, show local variations that allowed Fornasier et al. (2015) to distinguish three groups of physiographic regions (El-Maarry et al. 2015, 2016) that are characterized by low, medium, and high spectral slopes, respectively. The regions with relatively bluer (i.e., lower) spectral slopes correspond to brighter regions enriched in water ice (e.g., Hapi), those with redder (i.e., higher) spectral slopes characterize darker regions with dust coatings (e.g., Ash), while the intermediated slopes are characterized by collapsed materials (e.g., Aten). However, the analysis of the spectral slope variations highlighted that there is no correlation between groups of spectral slopes and particular morphologies, and the spectral slopes are not representative of a vertical diversity in the composition of the nucleus (Fornasier et al. 2015). 
Table 1. OSIRIS-NAC image datasets.

\begin{tabular}{|c|c|c|c|c|c|c|c|}
\hline Activity & Date, time & Filters & Dataset & $\begin{array}{c}\alpha_{F 27} \\
\left(^{\circ}\right)\end{array}$ & $\begin{array}{l}\Delta_{F 27} \\
(\mathrm{~km})\end{array}$ & $\begin{array}{l}\operatorname{Res}_{F 27} \\
\left(\mathrm{mpx}^{-1}\right)\end{array}$ & $\begin{array}{l}\text { Physiographic } \\
\text { regions }\end{array}$ \\
\hline МТP026P/ & 2016-02-10, & $16(360.0) 24(480.7) 23(535.7)$ & \multirow[t]{3}{*}{$\mathrm{C}$} & \multirow[t]{3}{*}{65.76} & \multirow[t]{3}{*}{51} & \multirow[t]{3}{*}{0.95} & Wosret, Bastet, \\
\hline STP095/COLOR_ & 07:05-07:07 & $22(649.2) 27(701.2) 28(743.7)$ & & & & & Neith, Maftet, \\
\hline MAP_001 & & $41(882.1) 61(931.9) 71(989.3)$ & & & & & Anuket \\
\hline MTP025P/ & 2016-01-27, & $16(360.0) 24(480.7) 23(535.7)$ & \multirow[t]{3}{*}{$\mathrm{D}$} & \multirow[t]{3}{*}{62.24} & \multirow[t]{3}{*}{69} & \multirow[t]{3}{*}{1.30} & Wosret, Bastet, \\
\hline STP093/SHAP7a & $21: 22-21: 24$ & 22(649.2) 27(701.2) 28(743.7) & & & & & Neith, Maftet, \\
\hline & & 41(882.1) 61(931.9) 71(989.3) & & & & & Anuket \\
\hline
\end{tabular}

Notes. The second and third columns refer to the date, time span, and filter number (with the relative central wavelength in nm) of the image acquisitions. The image sequences were used to obtain multispectral images renamed datasets C and D. Columns $\alpha_{F 27}, \Delta_{F 27}$, and Res ${ }_{F 27}$ refer to the phase angle, the distance between the spacecraft and the comet, and the image spatial resolution. The last column reports the framed physiographic regions.

The Visible and InfraRed Thermal Imaging Spectrometer (VIRTIS, Coradini et al. 2007) observations indicate a global surface composition dominated by dehydrated and organic-rich refractory materials (Capaccioni et al. 2015), although several local bright spots were interpreted as exposures of water ice (Pommerol et al. 2015; Fornasier et al. 2016; Filacchione et al. 2016). Overall, the sublimation processes are influenced by the different diurnal insolation conditions, by the proximity to perihelion passage (i.e., seasonal variations), and by the different morphologies of the cometary nucleus that lead to regional variations of the local volatile content (Vincent et al. 2015). Some regions are indeed subject to cyclical sublimation processes as a function of the illumination conditions over the local day. In this case, the activity is only partially connected to the exposure of ices and mainly depends on a sort of daily water cycle that affects the uppermost layers (De Sanctis et al. 2015). Approaching perihelion, the increasing level of activity partially removes the overlying refractory mantling material. This causes the exposure of the underlying ice-rich layers and leads to bluer colors for the nucleus of 67P, with a decrease in the mean visible to near-infrared spectral slope up to $-30 \%$ and considerable diurnal color variations (Fornasier et al. 2016), as well as a shortward shift of the VIRTIS $3200 \mathrm{~nm}$ absorption band (Longobardo et al. 2019, and references therein).

The layering of the consolidated material is persistent at the regional scale and can be easily recognized on the systems of terraces and cliffs, cuestas, and mesas that characterize the morphology of the comet (Massironi et al. 2015). Belton et al. (2018) suggested that this well-organized system of strata is due to the modification of the primitive cometary material. Specifically, they suggested that it is the result of the deep inward propagation of water-ice phase-change fronts that alternating active and quiescent phases led to the formation of intra-strata materials and strata boundaries, respectively.

The different orientation of the terraces was measured by Penasa et al. (2017) to produce a three-dimensional ellipsoidbased geometrical model (ellipsoidal model, EM) with which the intersection of the nucleus layering with the topography can be predicted. This model can be used to define the position of any point on the cometary nucleus in terms of its structural elevation, which provides a distance measure from the center of the ellipsoidal layering (i.e., points at same structural elevation lie on the same ellipsoid-shaped layer). The model was used by Ferrari et al. (2018) to verify the spectrophotometric variegation of the layering of the large lobe of $67 \mathrm{P}$ as a function of the structural elevation. The EM allowed them to distinguish two distinct classes of the outcropping nucleus material by means of a maximum likelihood (ML) supervised classification. This classification highlighted a correlation between the absolute reflectance and the structural elevation of the material. The two proposed classes show a different brightness and spatial distribution in function of the EM: the inner class is brighter and localized on the innermost layers (i.e., Imhotep region), and the outer class is darker and localized on the outermost layers (i.e., Apis region). This spectrophotometric variegation can have been enhanced by localized volume removals that led to the exposure of an inner shell that is brighter than the outer shell. Following their interpretation, the surface brightness variegation can be attributed to a difference in composition (e.g., different content in volatile materials) or textural properties (i.e., roughness and/or porosity) of the cometary nucleus.

In this work, we investigate the spectrophotometric properties of the small lobe layering of 67P by means of images acquired by the OSIRIS NAC camera. These images include the southern regions Wosret, Bastet (southern portion) and Neith and part of the northern regions Maftet and Anuket (El-Maarry et al. 2015, 2016). The southern hemisphere of 67P appears flattened and topographically less dynamic than the northern hemisphere, showing lesser textural diversity and the lack of large dust-covered regions, wide-spread smooth terrains, and large depressions (Lee et al. 2016). On the basis of the EM, these regions represent an extended geological section of the onion-like stratification of the small lobe, with the outermost layers outcropping in Bastet, Maftet and in the part of Wosret toward Hatmehit, whereas Neith, Anuket, and the part of Wosret toward the neck correspond to the innermost layers. The results obtained from the spectrophotometric analysis of the small-lobe layering were then integrated and compared with those obtained by Ferrari et al. (2018) for the large lobe.

\section{Geological setting}

The images used for this work were acquired with the OSIRIS NAC imaging system, which is equipped with 12 filters in the wavelength range between 250 and $1000 \mathrm{~nm}$ (NUV-VIS-NIR). The two selected image sequences (Table 1 and Figs. 1a and b) are post-perihelion acquisitions of January and February 2016 (2.44-2.33 au) that include the Wosret, Bastet, Neith, Maftet, and Anuket regions. They cover the maximum number of available filters (9 each) and have a similar spatial resolution $\left(0.95-1.3 \mathrm{~m} \mathrm{px}^{-1}\right)$ and phase angles $\left(62-65^{\circ}\right)$. 

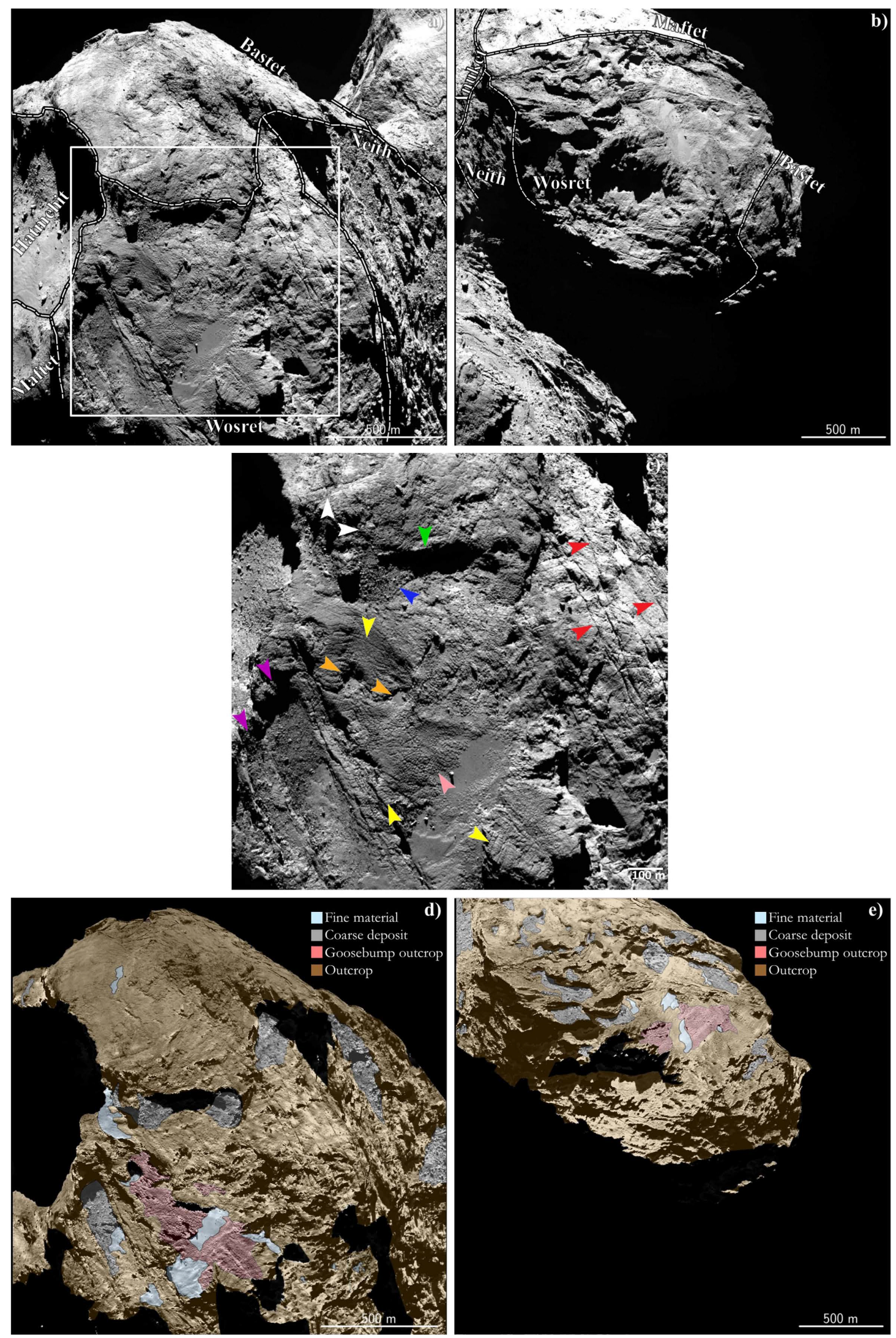

Fig. 1. View of the small lobe of 67P with subdivision into physiographic regions: (a) NAC_2016-02-10T07.06.04.905Z_IDG0_1397549400_F22 and (b) NAC_2016-01-27T21.22.31.724Z_IDG0_1397549000_F22, displayed in Rosetta standard orientation with the sun at the top. (c) Close-up of the southern hemisphere of the small lobe. The boundary between Wosret and Bastet is marked by a scarp (indicated by a green arrow) and debris with boulders (indicated with a blue arrow). Ochre arrows point to pits on Wosret, and the white arrows highlight a difference in tone between the northern (brighter) and southern (darker) portion of Bastet. The purple arrows indicate two spaced bands of layered material. Red and yellow arrows point to fractures, and the pink arrow highlights polygonal cracks. $(d)$ and $(e)$ Geomorphological maps realized on images (a) and (b), respectively. 
Table 2. Root mean square error (RMSE) warping values.

\begin{tabular}{cll}
\hline \hline Dataset & Filters & RMSE \\
\hline $\mathrm{C}$ & $22-27$ & 1.39 \\
& $23-27$ & 1.06 \\
& $24-27$ & 0.76 \\
& $16-27$ & 0.55 \\
& $28-27$ & 0.47 \\
& $41-27$ & 0.77 \\
& $61-27$ & 1.30 \\
& $71-27$ & 1.66 \\
\hline $\mathrm{D}$ & $22-27$ & 0.35 \\
& $23-27$ & 0.41 \\
& $24-27$ & 0.41 \\
& $28-27$ & 0.39 \\
& $41-27$ & 0.36 \\
& $71-27$ & 0.41 \\
& $61-27$ & 0.41 \\
& $16-27$ & 0.49 \\
\hline
\end{tabular}

In general, the regional units in the southern regions of 67P are fractured consolidated terrains, in which it is possible to observe evidence of stratification, and loose unconsolidated materials that consist of talus and gravitational accumulation deposits with isolated boulders (El-Maarry et al. 2016; Lee et al. 2016; Pajola et al. 2016).

Most of the southern hemisphere on the small lobe of 67P lies in Wosret region, which appears topographically flat, with some great changes of relief near the boundary with Maftet and Hatmehit regions. It is also characterized by outcrops of consolidated terrains that are dominated by fractures, together with pitted areas and polygonal cracks (e.g., close to Maftet and the Hatmehit depression. These are indicated in Fig. 1c with yellow, ochre, and pink arrows) (Lee et al. 2016; Auger et al. 2018). Toward the northern Maftet region, which is defined by a higher plateau that encloses several dust-covered irregular depressions with boulders (El-Maarry et al. 2015), various almost parallel lineaments form two bands of layered material at a distance of about one hundred meters from each other (Fig. 1c, purple arrows), and the rough surface toward the neck is marked by several fractures that reach $300 \mathrm{~m}$ in length (Fig. 1c, red arrows). The boundary between Wosret and Bastet is instead characterized by a scarp (Fig. 1c, green arrow) with debris and boulders at its feet (Fig. 1c, blue arrow). Bastet is a rough consolidated region that straddles the northern and southern hemispheres of the small lobe of 67P. Compared to the northern part, the southern part is more fractured and appears darker (Fig. 1c, white arrows) (El-Maarry et al. 2015, 2016). El-Maarry et al. (2016) interpreted this different hue to the lack of lightertoned dust coatings. On the other side, Wosret borders the Anuket region, which is characterized by outcrops of consolidated material (Giacomini et al. 2016). Finally, the Neith region, located on the neck, exhibits strata heads that are cut by fracture systems and gravitational deposits at their feet (Lee et al. 2016).

\section{Methods}

In order to compare the morphological domains and the spectrophotometric properties of the cometary material, we made a geomorphological map for each set of images by means of the software Esri ArcGis 10.4.1 (Figs. 1d and e). The maps highlight that consolidated materials are present throughout, and evidence of the stratification is ubiquitous. We also distinguish some unconsolidated material units: coarse deposits and fine materials. The first includes mass-wasting deposits and boulder fields that may have been generated by sublimation processes and gravitational collapses. The fine materials include areas that are characterized by smooth dust coatings, which are potentially due to airfall deposition and can be easily transferred from a site to another. We then converted the images of Table 1, which are calibrated in spectral radiance (expressed in $\mathrm{W} \mathrm{m}^{-2} \mathrm{~nm}^{-1} \mathrm{sr}^{-1}$ ) according to the OSIRIS standard calibration pipeline (Tubiana et al. 2015), to reflectance (i.e., radiance factor I/F) using the following equation:

$\frac{I}{F}=\frac{\pi r_{\mathrm{h}}^{2} I_{\text {meas }}}{F_{\mathrm{s}}}$,

where $I_{\text {meas }}$ is the measured spectral radiance, $F_{\mathrm{s}} / \pi$ is the solar spectral irradiance at 1 au measured at the central wavelength for each filter, and $r_{\mathrm{h}}$ is the heliocentric distance of the comet in au. Successively, the images of each sequence were reorganized according to the filter wavelengths and were coregistered through at least 40 ground control points (GCPs) for each image using the image-to-image function of the software Exelis Envi Classic 5.0 and using the WARP_TRI function (i.e., linear interpolation method) of the software Exelis IDL 5.0. The root mean square errors (RMSEs) resulting from the GCP positioning are listed in Table 2. Two multispectral images framing the areas of interest were thus created and renamed datasets $\mathrm{C}$ and $\mathrm{D}$, consecutively to datasets A and B by Ferrari et al. (2018).

We used the stereo-photoclinometry shape model SHAP8_v1.9 of comet 67P by Jorda et al. (2016) combined with the NAIF-SPICE kernels and relative IDL code toolkit (Acton 1996; Acton et al. 2018) to produce synthetic images that reproduce the original illumination conditions of the comet. This allowed us to derive the illumination angles at the moment of image acquisition and to exclude emission and incidence angles greater than $80^{\circ}$ from the processing.

Finally, to correct the illumination and topographic effects, we applied the Akimov disk function (Shkuratov et al. 2011, and references therein), and in particular, its parameter-free version. As tested by La Forgia et al. (2015), the Akimov parameterless function works well on the surface of 67P and indeed yields the best approximation to the Hapke correction, with the advantage that it has no parameters. In addition, for the purpose of this study, we did not need to derive an absolute albedo, and given the small phase angle coverage of our data, it is sufficient to retrieve the equigonal albedo at phase angles $62-65^{\circ}$. Because residual values due to small misalignments of the filters were still present in shadowed areas, we brought the signal of all the pixels with reflectance values lower than 0.002 to background value. Falsecolor images obtained by the multispectral images are shown in Figs. $2 \mathrm{a}$ and $\mathrm{b}$.

The obtained nine-filter multispectral images were then used to produce spectral slope maps, for which the following equation was used:

$S=\frac{I / F_{\text {NearIR }}-I / F_{\text {Green }}}{\lambda_{\text {NearIR }}-\lambda_{\text {Green }}} \cdot \frac{10^{4}}{I / F_{\text {Green }}}$,

where $I / F_{\text {NearlR }}$ and $I / F_{\text {Green }}$ are the Akimov-corrected reflectances for the near-infrared (F41, $882.1 \mathrm{~nm})$ and green $(\mathrm{F} 23,535.7 \mathrm{~nm})$ filters, and the slope is expressed in percent $/ 100 \mathrm{~nm}$. Figures $2 \mathrm{c}$ and d show the spectral slope maps for the two datasets. 

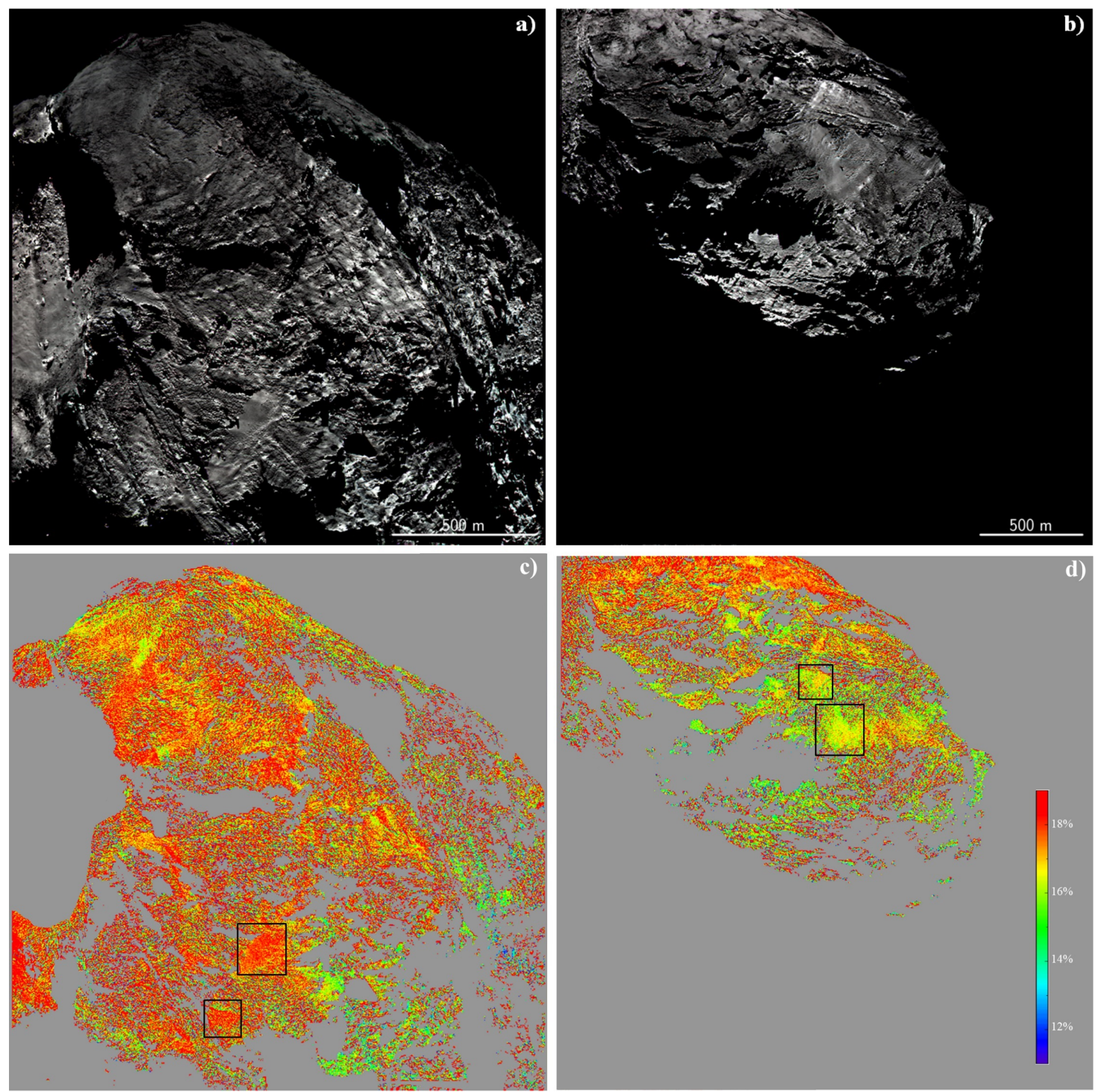

Fig. 2. Panels $a$ and $b$ : false-colors images (red, green, and blue correspond to filters 28,23 , and 24 , centered at $743.7 \mathrm{~nm}, 535.7 \mathrm{~nm}$, and $480.7 \mathrm{~nm}$, respectively) obtained by the multispectral images. Panels $c$ and $d$ : spectral slope maps for the two datasets obtained with Eq. (2); the black boxes frame the same areas of fine material deposits; they show different spectral slope values.

We decided to perform a first two-class ML supervised classification on the multispectral images by means of the software Exelis Envi Classic 5.0, with the aim to distinguish the consolidated outcrops from the fine material deposits. These deposits might be non-autochthonous of the regions under study because they may have been deposited by airfall. This makes them unsuitable for determining the spectral behavior of the stratification. Conversely, we combined the consolidated outcrops with their related coarse deposits, which, being present near scarps and terraces, can be considered as autochthonous mass-wasting deposits generated by sublimation processes and gravitational collapses. Dataset C frames a portion of the Hatmehit depression, which presents a wide deposit of fine materials that are probably due to airfall deposition, therefore we decided in advance to exclude it from the classifications, along with the framed portions of the large lobe of 67P.

To perform an ML supervised classification, the guidelines have to be defined. These are regions of interest (ROIs) that the software must follow to attribute pixels to one class or another on the basis of the likeness to the given datum. Therefore, we selected a ROI that was representative of the consolidated and coarse deposits and a ROI that was representative of the fine 

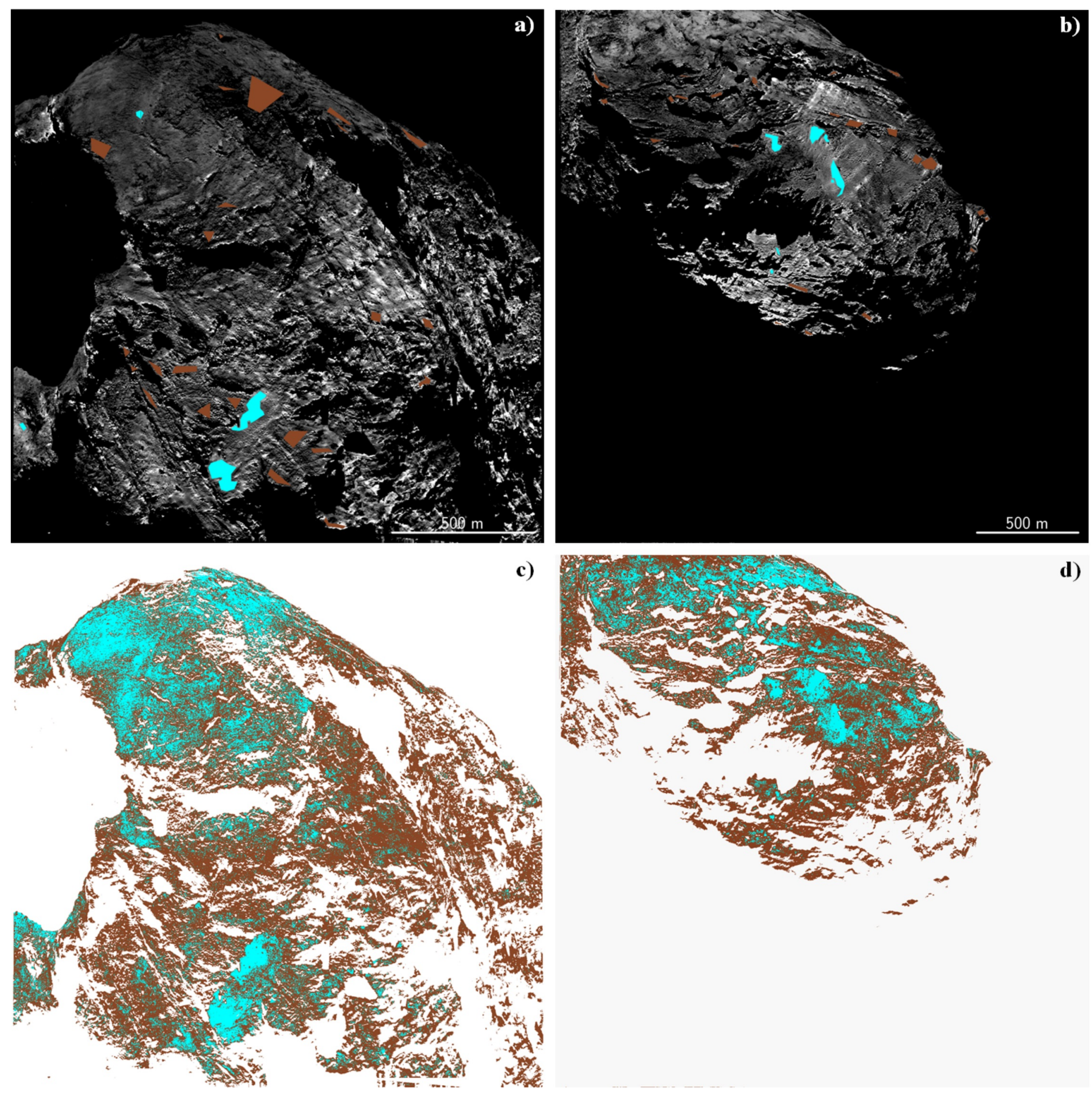

Fig. 3. Panels $a$ and $b$ : multispectral-image filter 27 centered at $701.2 \mathrm{~nm}$ overlapped by polygonal ROIs corresponding to fine materials (cyan) and consolidated materials (brown) created on the basis of the geomorphological maps of Figs. $1 \mathrm{~d}$ and e. Panels $c$ and $d$ : maximum likelihood supervised classification of fine materials (cyan class) and consolidated materials (brown class).

materials by grouping pixels on the basis of the previously made geomorphological maps (Figs. 3a and b).

By comparing the results of the classifications (Figs. 3c and d) with the geomorphological maps (Figs. 1d and e), it is possible to verify the overall agreement in terms of class distributions. The cyan class fully identifies the mapped fine material deposits (i.e., light blue unit), allowing us to also infer an extremely thin dust coating on the outcropping Bastet region. The class corresponding to the outcropping consolidated terrains and their relative deposits (i.e., brown class) covers the entire remaining surface.
After they were identified, the pixels related to the fine material classes were excluded in order to obtain two multispectral images that ideally were made up by the outcropping consolidated materials and their coarse mass-wasting deposits alone (Figs. 4a and b).

To characterize the spectrophotometric properties of the stratification of the small lobe, we then created new ROIs on the isolated outcropping material alone on the basis of the structural elevation defined by the EM (Penasa et al. 2017). ROIs corresponding to the outmost layers were defined in the Wosret and Bastet regions at a small-lobe structural elevation of 

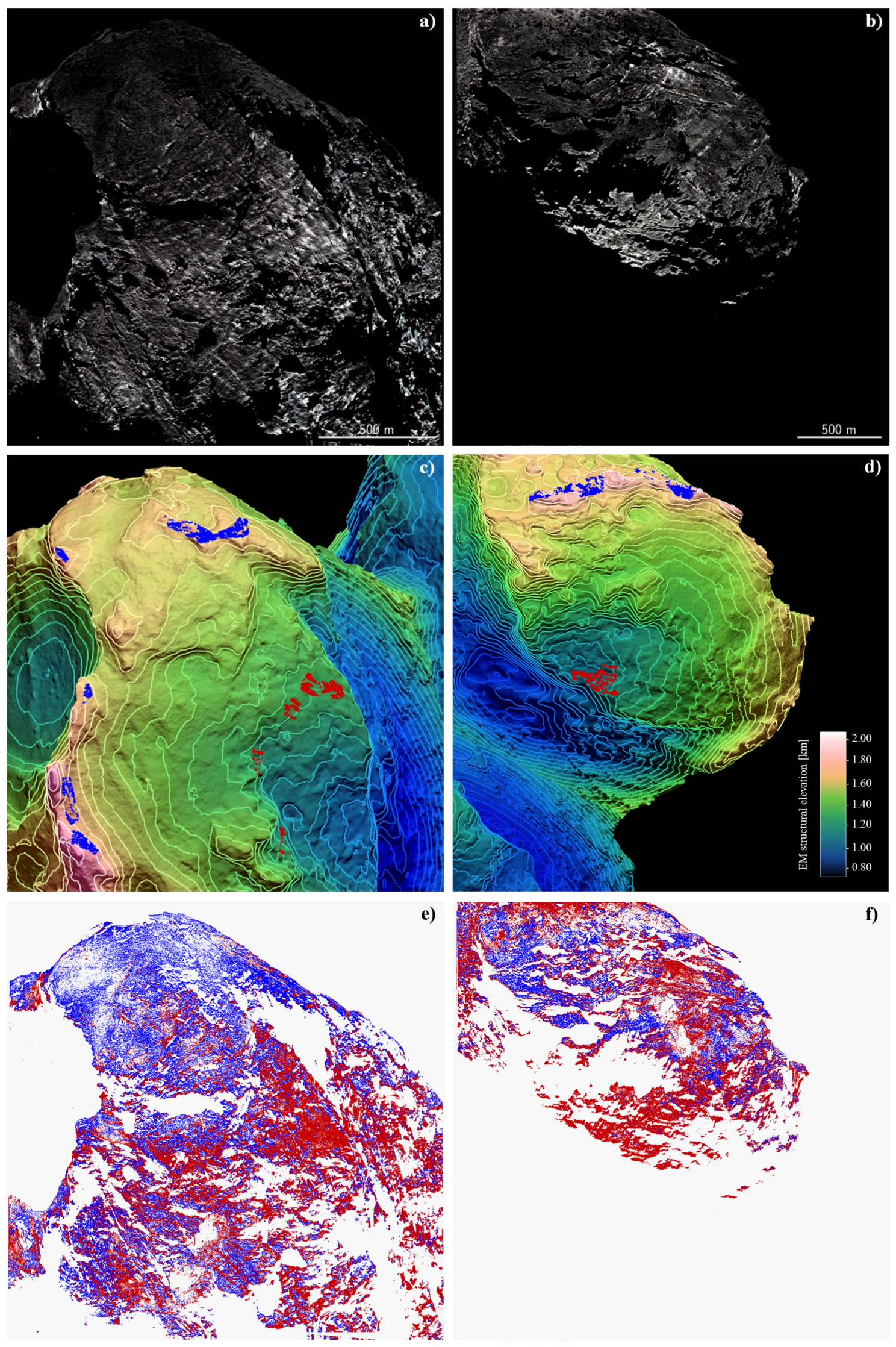

f)

Fig. 4. Panels $a$ and $b$ : false-color images (red, green, and blue correspond to filters 28,23 , and 24 centered at $743.7 \mathrm{~nm}, 535.7 \mathrm{~nm}$, and $480.7 \mathrm{~nm}$, respectively) obtained from multispectral images for both datasets in which the fine materials have been masked. Panels $c$ and $d$ : elevation model overlapped by the ROIs that were created according to structural elevation: red and blue ROIs correspond to the inner and outer class, respectively. Panels e and $f$ : maximum likelihood supervised classification performed on consolidated materials and coarse deposits. Red and blue boxes identify the inner and outer class, respectively. 
Table 3. Class divergence values of outer- and inner-ROI pixels.

\begin{tabular}{ccccc}
\hline \hline Dataset & Outer-ROI pixels & Inner-ROI pixels & Jeffries-Matusita & Transformed divergence \\
\hline C & 7690 & 10968 & 1.99 & 2.00 \\
\hline D & 5347 & 4333 & 1.98 & 2.00 \\
\hline
\end{tabular}

Notes. Class divergence values are calculated through the Jeffreis-Matusita and transformed divergence measurements for both datasets.

$1.65-1.80 \mathrm{~km}$ (shown in blue in Figs. 4c and d), while ROIs corresponding to the innermost layers were defined in the part of Wosret toward the neck region at an elevation of about 1.20 $1.45 \mathrm{~km}$ (shown in red in Figs. $4 \mathrm{c}$ and d). Elevation ranges were chosen so that the ROIs grouped well-represented but noncontiguous outcrops. After we defined them, we verified the spectral separability (i.e., good class divergence) of these ROIs by means of the Jeffries-Matusita and transformed divergence separability measurements (Table 3) (Richards \& Jia 2006). They both give an exponentially decreasing weight to increasing separations between spectral classes. A good class divergence is reached when these parameters are asymptotic to 2.00. Finally, we used these ROIs to perform a new ML supervised classification of the surface of the small lobe, defining an outer and an inner class, which are representative of the outermost and innermost packs of layers (Figs. 4e and f).

\section{Results}

Geomorphological maps based on datasets $\mathrm{C}$ and D highlight that most of the southern hemisphere of the small lobe of $67 \mathrm{P}$ is mainly characterized by outcropping consolidated terrains with large fractured areas and accumulations of coarse deposits that are caused by the detachment of material through sublimation and erosion; only a few areas present smooth and fine material deposits.

The datasets used in this work provide spectral slope values of about $16-18 \%$ at phase angles of $62-65^{\circ}$ over most of the available cometary surface (Figs. $2 \mathrm{c}$ and d). This is consistent with values obtained by Fornasier et al. (2016) in the same area. The largest variability has been measured on the dustcovered terrains, which are not as spread out in this portion of the comet. They present values between 15 and $18 \%$. In particular, some fine-material deposits show different spectral slope values depending on the dataset. The two largest deposits of dataset $\mathrm{C}$ display a spectral slope of about $18 \%$, whereas in dataset $\mathrm{D}$, the same deposits have slopes ranging from 15 to $17 \%$ (Figs. 2c and d black boxes).

Results of the two-class ML supervised classification that we applied to the consolidated outcrops and their relative coarse deposits are shown in Figs. 4e and f. The inner class is mainly localized at the low EM elevations of the Neith, Anuket, and Wosret regions, whereas the outer class is instead more representative of the high EM elevations of the Bastet, Maftet, and Wosret regions (see Figs. $4 \mathrm{c}$ and d).

Concerning the spectral properties, the absolute average spectrum pertaining to the inner class presents a systematically higher reflectance than the outer class (Fig. 5). Notably, very similar pixels can have been attributed to different classes in accordance to their maximum likeliness to one ROI rather than to the other. This of course provides large error bars for the average class spectra. The average spectral reflectance of the outer classes ranges from $0.3 \%$ at $360 \mathrm{~nm}$ to $0.9 \%$ at $989 \mathrm{~nm}$ in both datasets, while the inner classes reach average reflectances that are $80-90 \%$ and $60-65 \%$ greater than the outer class values for datasets $\mathrm{C}$ and $\mathrm{D}$, respectively. Moreover, although the selected datasets were acquired five months after perihelion passage, that is, when sublimation was notably reduced, the absolute spectral slopes of both classes display an enhancement of the flux in the 700-750 nm wavelength range. Reflectance values were then normalized to the green wavelength $(535.7 \mathrm{~nm})$, in agreement with most of the literature on primitive solar system bodies (e.g., Barucci et al. 2005). In this way, we obtained nearly identical slopes for all the classes, with the exception of the wavelength ranges larger than $900 \mathrm{~nm}$, where the slope of the inner class slightly decreases with respect to the slope of the outer class for both datasets (Fig. 5). However, when we take the absolute values into account, the differences are always within the error bars. Comparing the values for the different classes and the results we obtained for the two datasets, we conclude that the main distinguishing factor for the assignment of the pixel to a specific class is the absolute reflectance, not the spectral slope.

\section{Discussion}

We have shown that the spectrophotometric investigation that was performed on OSIRIS-NAC multispectral datasets that frame large parts of the small lobe of $67 \mathrm{P}$ has determined values for the spectral slope that range between 16 and $18 \%$ in the VIS-NIR wavelength range. Moreover, the supervised classifications we applied to the datasets that only consist of data pertaining to outcrops and relative coarse deposits showed that layers that are located at higher structural elevations have a lower brightness than the innermost layers. We now proceed by comparing (i) the results of the two datasets of the small lobe of 67P (i.e., datasets $\mathrm{C}$ and D) and then (ii) these results with those obtained by the analysis of the large lobe described in Ferrari et al. (2018).

Overall, the spectral slope maps highlight that after perihelion passage, most of the cometary surface has mean spectral slope values that lie in the interval $16-18 \%$ at phase angles of $62-65^{\circ}$. The fine-material deposits range between 14 and $18 \%$ and some ice-rich patches are still visible on the large lobe. That the values for dataset $\mathrm{C}$ are somewhat higher than those for dataset D (Figs. 2c and d black boxes) might be described, for example, to a decrease in volatile content or to the different local solar time because dataset C (10 February 2016) was acquired two weeks after dataset D (27 January 2016) and at a different a sub-solar longitude $\left(53.6^{\circ}\right.$ for dataset $C$ and $292.6^{\circ}$ for dataset D). In general, the spectral slope ranges we obtained for the geomorphological domains of the small lobe are consistent with those of the large lobe.

The ML classifications restricted to the consolidated outcrops and the associated coarse deposits were finalized to highlight possible different characteristics depending on the surface structural elevation and allowed us to associate these materials to either the inner or the outer class. For all the datasets 

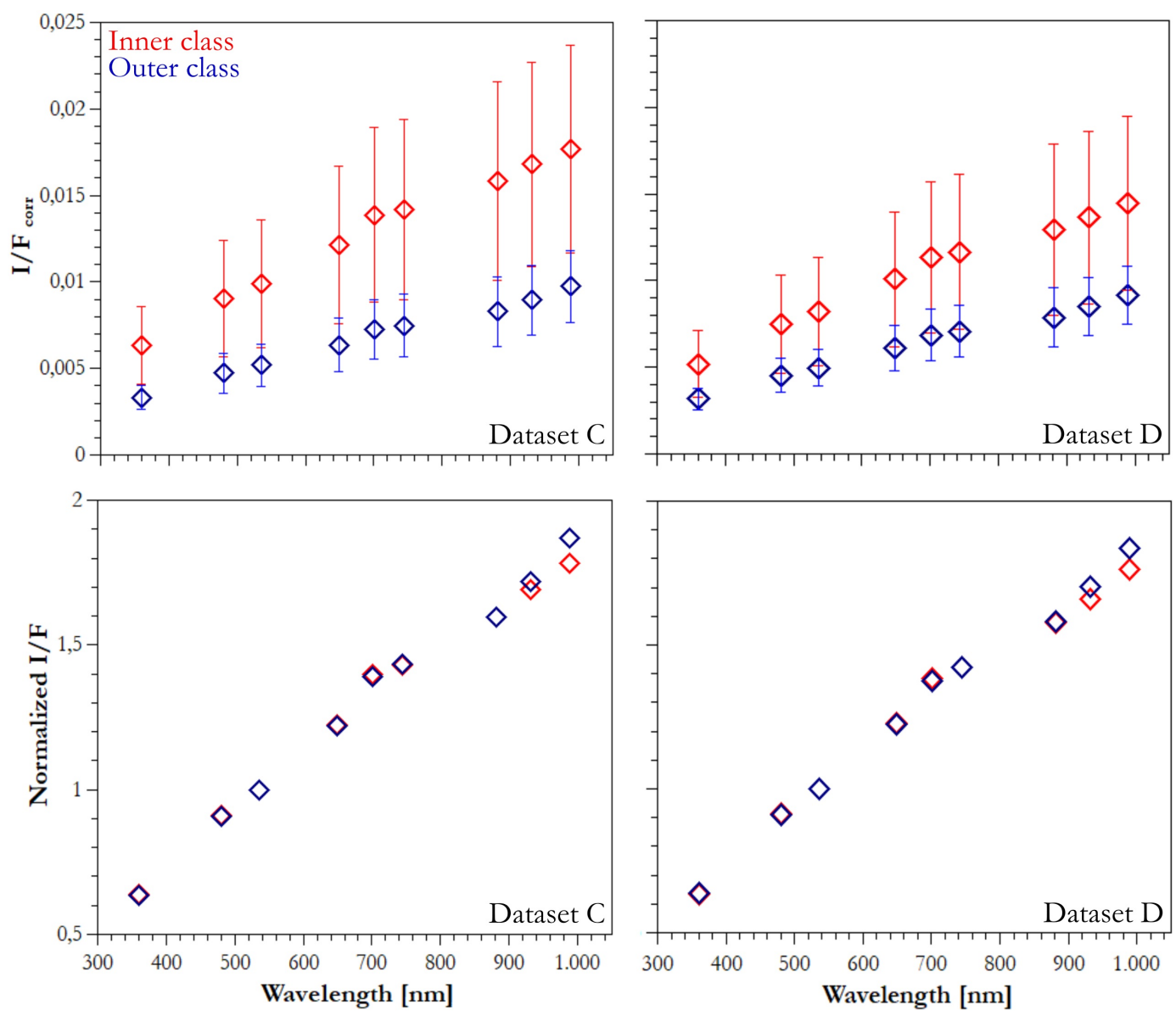

Fig. 5. Absolute (upper panels) and normalized (bottom panels) average spectra of inner and outer classes for datasets C and D.
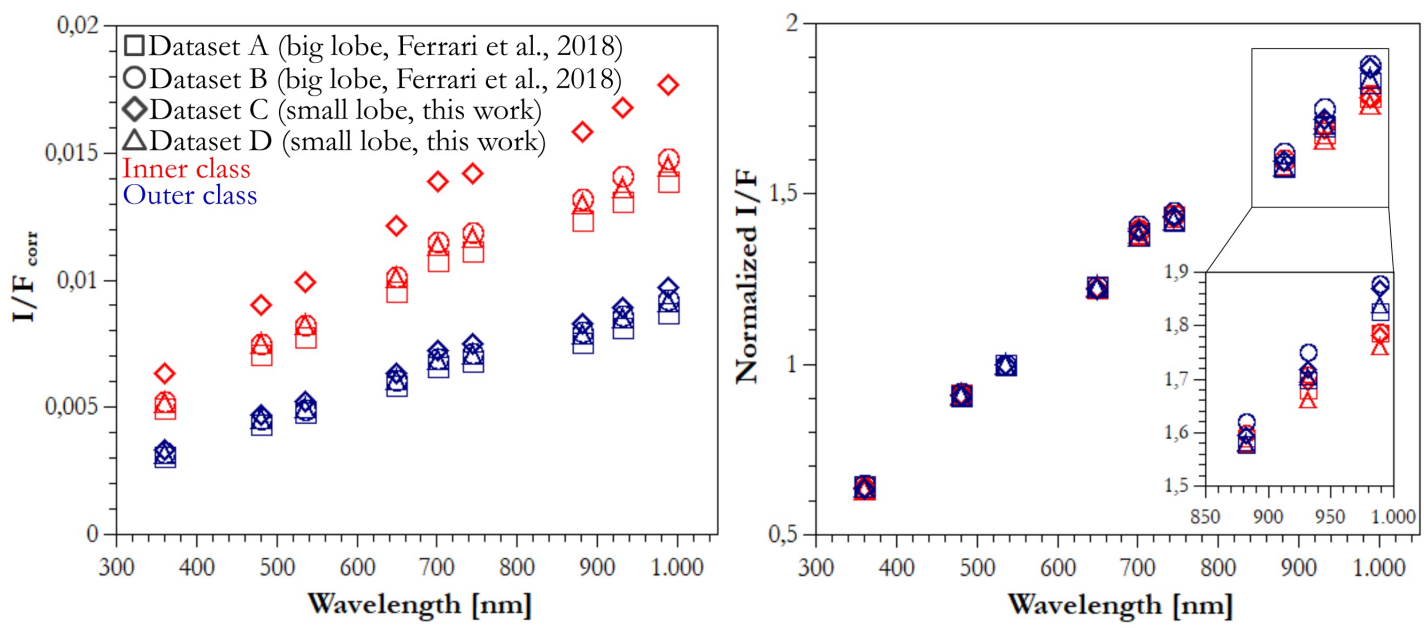

Fig. 6. Comparison of absolute (left panel) and normalized (right panel) average spectra of inner and outer classes for datasets A, B, C, and D.

(Ferrari et al. 2018, and this study), we verified that the two classes are reasonably closely associated with the structural elevation of the lobe, and that the distinctive characteristic between the two is the absolute reflectance: on both lobes, the innermost are brighter than the outermost outcrops. The spectral surface reflectance of the outer classes has similar values on both lobes: they range from about $0.3 \%$ at $360 \mathrm{~nm}$ up to about $0.9 \%$ at $989 \mathrm{~nm}$. The absolute reflectance of the inner classes for datasets $\mathrm{A}, \mathrm{B}$, and $\mathrm{D}$ ranges from $0.5 \%$ to about $1.4 \%$. The reflectance of dataset $\mathrm{C}$ of the inner class instead is $30 \%$ greater than that of the others (Fig. 6, left panel). However, the brightness variation between outer and inner classes is not associated with a significant spectral variation: when it was normalized, only a minor decrease of the slope in the 900-1000 $\mathrm{nm}$ range is observed for inner classes (Fig. 6, right panel). The persistence of a bluer slope in the normalized average spectra at the nearinfrared wavelengths for the inner class might suggest that the innermost layers have a slightly different composition.

The brightness separation between the two classes can be associated with differences in the texture and/or composition 

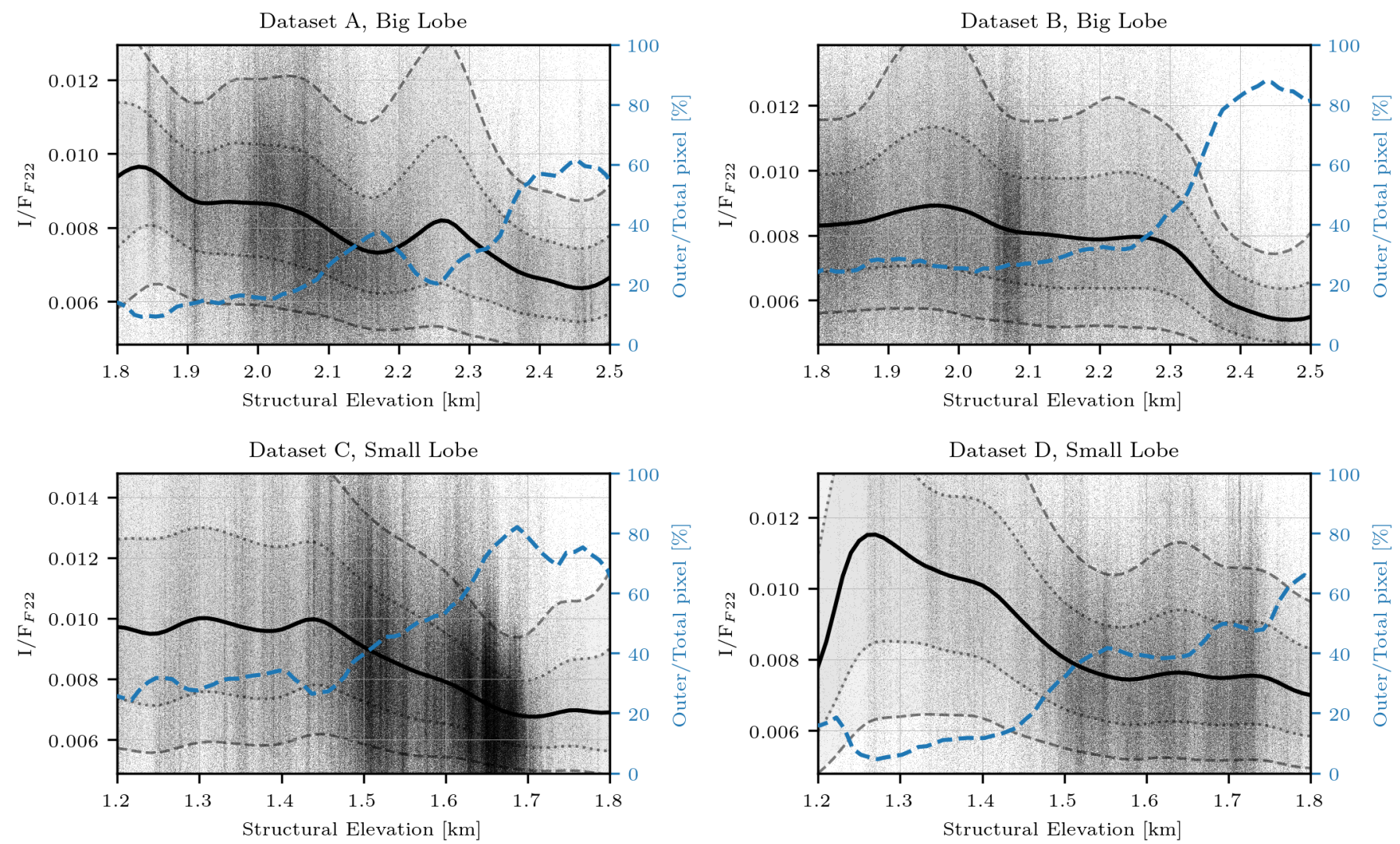

Fig. 7. Comparison between dataset results. Image datasets A, B, C, and D correspond to phase angles of $62.90,62.67,65.76$, and $62.24^{\circ}$ respectively. Large-lobe datasets A and B (Ferrari et al. 2018) are plotted in the top row, and small-lobe datasets C and D (this study) are plotted in the bottom row. For each plot, the blue dashed line shows the percentage of pixels that are classified as outer class with respect to the total number of classified pixels. Black dots indicate pixel values of filter $22(649.2 \mathrm{~nm})$ and the respective positions in the EM. The black solid line represents the running median of I/F values. Dotted lines enclose the 30.0-70.0 percentiles for the reflectance data, and the dashed lines enclose the 15.984.1 percentiles. Percentage curves and running medians were computed by binning the data $(0.05 \mathrm{~km}$ bins $)$ and then computing corresponding descriptive statistics for each bin.

(i.e., refractory and icy material content) of the consolidated cometary material. This also raises the question whether the variegation is primordial or evolutionary, that is, whether it occurred during the accretionary processes that independently involved the two lobes during their journey in the protoplanetary disk, or during the following evolutionary processes of the bilobed nucleus.

We then plotted the percentage of pixels belonging to each class as a function of their structural elevation (Fig. 7) for largelobe (Ferrari et al. 2018) and small-lobe data (this study). We verified that like for the large-lobe datasets (Fig. 7 top row), when we increase the structural elevation of the small lobe, the outer class proportionally increases with respect to the inner class (see Fig. 7 bottom row). This correlation demonstrates that layering is well identified by the classification.

From Ferrari et al. (2018), we know that a sharper increase in outer-class pixels to the detriment of the inner-class ones occurs in the large lobe at a structural elevation of about $2.2-2.3 \mathrm{~km}$ (Fig. 7 top row). This elevation is not reached on the small lobe. A similar kink, even at different structural elevations, is not so clearly observable in small-lobe data (Fig. 7 bottom row). However, the small lobe displays an overall increase in outerclass pixels toward higher elevations, similarly to the large lobe. Therefore, outcropping consolidated materials tend to be darker with increasing elevation of both lobes. This trend is also observable in the spectrophotometrically corrected $\mathrm{I} / \mathrm{F}$ values of the orange filter (F22, 649.2 nm) of all four datasets, which we plot as a function of the EM structural elevation (Fig. 7). Reflectance values display a gradual decrease in brightness toward higher elevations in all the datasets.

The progressive increase in darker materials toward higher elevations on both lobes agrees with a primordial as well as an evolutionary variegation. As described in Ferrari et al. (2018), a single-lobe variegation that occurred during accretion could be due to a different size or composition of the constituents and/or to their compaction (e.g., Davidsson et al. 2016). To date, we do not know the primitive (e.g., pre-junction) size and shape of each lobe and their accretion histories, and we have had little very localized access to the pristine material of 67P. Therefore, any detailed comparison of reflectance values as a function of the structural elevation between the two lobes here would be considered inconclusive for a primordial variegation.

For what concerns the possible evolutionary processes, the brightness variegation could be associated to the maturation and aging of the surface that is caused by particle radiation when the comet was at large heliocentric distances (Maquet 2015), and by insolation during closer perihelion passages. The maturation in this case acts on the texture as well as on the relative composition of the involved cometary volume. The maturation of exposed layers due to insolation, for instance, has been tested on analog samples under simulated cometary surface conditions. The experiments highlighted that the reflectance of the samples primarily depends on the composition, amount, and mixing mode of the refractory organic grains within the ice particles 
(Pommerol et al. 2015). Mixtures subjected to thermal conditioning in a comet-like environment acquire a strong vertical stratification, with an uppermost porous dark mantle of desiccated refractory dust overlaying an ice-rich subsurface formed by sintering and/or recondensation of water vapor (e.g., Grün et al. 1991).

On the comet, such modifications of texture and volatile content are thought to occur on volumes that undergo specific thermal variations, and they presumably do not exceed the meterto-decameter scale (Kossacki et al. 1997; Filacchione et al. 2016). At the same scale, a variegation of the outer material as a function of the depth has been observed for another comet: Sunshine et al. (2007) reported that below the first meter of dehydrated thermally processed material of 9P/Tempel, the content of ice particles appears to increase with increasing depth. In addition, in the short term, insolation rates are not uniform at the same structural elevation. On the same lobe, two areas located at the same structural elevation but on different hemispheres have not undergone the same insolation. In the long term, however, outer layers have presumably been exposed for longer than the internal layers. Concurrently, the detection of several jet sources (Vincent et al. 2016; Fornasier et al. 2017, 2019; Rinaldi et al. 2018, and references therein, this issue) and bright spots (Fornasier et al. this issue) in regions located at lower EM structural elevations (e.g., Khonsu, Anhur, Imhotep, Hapi, and Anuket) suggests that inner layers are currently more prone to local erosion and rejuvenation of the surface, and that outer layers could have completed such modification processes and now appear darker. Therefore, the progressively brighter deeper layers suggest that lobes might have accreted in a similar environment and with a gradual change of the constituent properties, and/or that layers were later modified through gradual exposition.

\section{Conclusions}

With the aim to spectrophotometrically investigate the layered surface of the small lobe of 67P, two sequences of images acquired by the OSIRIS Narrow Angle Camera were selected. The images, which are post-perihelion acquisitions with similar resolution and phase angle, were converted into reflectance and were photometrically corrected in order to obtain two multispectral images. For both datasets, we geomorphologically mapped autochthonous and allochthonous materials. The latter, that is, fine materials that are easily subjected to airfall deposition, were then masked, and a maximum likelihood supervised classification was performed on the outcropping consolidated material and relative coarse deposits. The classification allowed us to identify two distinct classes, the inner and outer class, which are characterized by different brightness, structural elevation, and spatial distribution. In particular, the outer class has lower reflectance values, is localized at higher structural elevations, and tends to decrease toward lower structural elevations to the advantage of the brighter inner class. This highlights a gradual darkening trend from lower toward higher elevations. These results are consistent with results that have been obtained for the large lobe.

The comparison of the results we obtained for the large and small lobes of 67P indicates that the main distinguishing factor that can be ascribed to the spectrophotometric variation is the brightness, which can be associated with a different surface texture and/or with a different composition of layers that are located at different elevation. The paucity of recent activity on the outer layers seems to suggest that their reservoirs are exhausted. This would imply that the brightness variegation is to a certain extent driven by the progressive maturation of the pre-existent layering, coupled with the periodic exposure of deeper fresh material of the nucleus of comet 67P. This scenario would be the result of an evolutionary process.

Acknowledgements. We are grateful to Andrea Longobardo for suggestions and corrections that improved our paper. OSIRIS was built by a consortium of the Max-Planck-Institut für Sonnensystemforschung, Göttingen, Germany, CISAS - University of Padova, Italy, the Laboratoire d'Astrophysique de Marseille, France, the Instituto de Astrofísica de Andalucia, CSIC, Granada, Spain, the Research and Scientific Support Department of the European Space Agency, Noordwijk, The Netherlands, the Instituto Nacional de Técnica Aeroespacial, Madrid, Spain, the Universidad Politéchnica de Madrid, Spain, the Department of Physics and Astronomy of Uppsala University, Sweden, and the Institut für Datentechnik und Kommunikationsnetze der Technischen Universität Braunschweig, Germany. The support of the national funding agencies of Germany (DLR), France (CNES), Italy (ASI), Spain (MEC), Sweden (SNSB), and the ESA Technical Directorate is gratefully acknowledged.

\section{References}

Acton, C. H. 1996, Planet. Space Sci., 44, 65

Acton, C., Bachman, N., Semenov, B., \& Wright, E. 2018, Planet. Space Sci., 150,9

Auger, A. T., Groussin, O., Jorda, L., et al. 2018, Icarus, 301, 173 Barucci, M. A., Belskaya, I. N., Fulchignoni, M., et al. 2005, ApJ, 130, 1291 Belton, M. J., Zou, X. D., Li, J. Y., \& Asphaug, E. 2018, Icarus, 314, 364 Capaccioni, F., Coradini, A., Filacchione, G., et al. 2015, Science, 347, aaa0628 Coradini, A., Capaccioni, F., Drossart, P., et al. 2007, Space Sci. Rev., 128, 529 Davidsson, B. J. R., Sierks, H., Güttler, C., et al. 2016, A\&A, 592, A63 De Sanctis, M. C., Capaccioni, F., Ciarniello, M., et al. 2015, Nature, 525, 500 El-Maarry, M. R., Thomas, N., Giacomini, L., et al. 2015, A\&A, 583, A26 El-Maarry, M., Thomas, N., Gracia-Berná, A., et al. 2016, A\&A, 593, A110 Ferrari, S., Penasa, L., La Forgia, F., et al. 2018, MNRAS, 479, 1555

Filacchione, G., De Sanctis, M. C., Capaccioni, F., et al. 2016, Nature, 529, 368 Fornasier, S., Feller, C., Lee, J. C., et al. 2017, MNRAS, 469, S93 Fornasier, S., Hasselmann, P. H., Barucci, M. A., et al. 2015, A\&A, 583, A30

Fornasier, S., Mottola, S., Keller, H. U., et al. 2016, Science, 2671, aag2671

Fornasier, S., Hoang, V. H., Hasselmann, P. H., et al. 2019, A\&A, 630, A7 (Rosetta 2 SI)

Giacomini, L., Massironi, M., El-Maarry, M. R., et al. 2016, MNRAS, 462, S352 Grün, E., Bar-Nun, A., Benkhoff, J., et al. 1991, in Comets in the Post-Halley Era, eds. J. R. L. Newburn, M. Neugebauer, \& J. Rahe (Dordrecht: Kiuwer Academic Publishers), 277

Jorda, L., Gaskell, R., Capanna, C., et al. 2016, Icarus, 277, 257

Keller, H. U., Barbieri, C., Lamy, P., et al. 2007, Space Sci. Rev., 128, 433

Kossacki, K. J., Kömle, N. I., Leliwa-Kopystyński, J., \& Kargl, G. 1997, Icarus, 128,127

La Forgia, F., Giacomini, L., Lazzarin, M., et al. 2015, A\&A, 583, A41 Lee, J. C., Massironi, M., Ip, W. H., et al. 2016, MNRAS, 462, S573 Longobardo, A., Della Corte, V., Ivanovski, S., et al. 2019, MNRAS, 483, 2165 Maquet, L. 2015, A\&A, 579, A78

Massironi, M., Simioni, E., Marzari, F., et al. 2015, Nature, 526, 402

Pajola, M., Oklay, N., La Forgia, F., et al. 2016, A\&A, 592, A69

Penasa, L., Massironi, M., Naletto, G., et al. 2017, MNRAS, 469, S741 Pommerol, A., Thomas, N., El-Maarry, M. R., et al. 2015, A\&A, 583, A25

Richards, J. A., \& Jia, X. 2006, Remote Sensing Digital Image Analysis: An Introduction (Berlin: Springer-Verlag), 439

Rinaldi, G., Bockelée-Morvan, D., Ciarniello, M., et al. 2018, MNRAS, 481, 1235

Shkuratov, Y., Kaydash, V., Korokhin, V., et al. 2011, Planet. Space Sci., 59, 1326 Sierks, H., Barbieri, C., Lamy, P. L., et al. 2015, Science, 347, aaa1044 Sunshine, J. M., Groussin, O., Schultz, P. H., et al. 2007, Icarus, 190, 284 Tubiana, C., Güttler, C., Kovacs, G., et al. 2015, A\&A, 583, A46

Vincent, J. B., Bodewits, D., Besse, S., et al. 2015, Nature, 523, 63 Vincent, J. B., A'Hearn, M. F., Lin, Z. Y., et al. 2016, MNRAS, 462, S184

1 Center of Studies and Activities for Space (CISAS) "G. Colombo", University of Padova, Via Venezia 15, 35131 Padova, Italy e-mail: sabrina.ferrari@unipd.it

2 Department of Physics and Astronomy "G. Galilei", University of Padova, Vicolo dell'Osservatorio 3, 35122 Padova, Italy

3 Department of Geosciences, via G. Gradenigo 6, University of Padova, 35131 Padova, Italy 
${ }^{4}$ Department of Physics and Astronomy "G. Galilei", University of Padova, via Marzolo 8, 35131 Padova, Italy

5 CNR-IFN UOS Padova LUXOR, Via Trasea 7, 35131 Padova, Italy

${ }^{6}$ INAF, Astronomical observatory of Padova, Vicolo dell'Osservatorio 5, 35122 Padova, Italy

7 Max Planck Institute for Solar System Research, Justusvon-LiebigWeg 3, 37077, Göttingen, Germany

8 Jet Propulsion Laboratory, M/S 183-401, 4800 Oak Grove Drive, Pasadena, CA 91109, USA

9 LESIA, Observatoire de Paris, Université PSL, CNRS, Université Paris Diderot, Sorbonne Paris Cité, Sorbonne Université, 5 Place J. Janssen, 92195 Meudon Principal Cedex, France

10 Deutsches Zentrum für Luft- und Raumfahrt (DLR), Institut für Planetenforschung, Rutherfordstraße 23, 12489 Berlin, Germany

11 Konkoly Observatory, PO Box 67, 1525 Budapest, Hungary

12 Laboratoire d'Astrophysique de Marseille, UMR 7326, CNRS \& Aix Marseille Université, 13388 Marseille Cedex 13, France

13 Centro de Astrobiologia, CSIC-INTA, 28850 Torrejon de Ardoz, Madrid, Spain

${ }^{14}$ International Space Science Institute, Hallerstrasse 6, 3012 Bern, Switzerland
${ }^{15}$ Science Support Office, European Space Research and Technology Centre/ESA, Keplerlaan 1, Postbus 299, 2201 AZ Noordwijk ZH, The Netherlands

${ }^{16}$ LATMOS, CNRS/UVSQ/IPSL, 11 Boulevard d'Alembert, 78280 Guyancourt, France

17 Physics Department, Auburn University, 206 Allison Laboratory, Auburn, AL 35849, USA

18 Department of Industrial Engineering, University of Padova, Via Venezia 1, 35131 Padova, Italy

${ }^{19}$ Faculty of Engineering, University of Trento, via Mesiano 77, 38121 Trento, Italy

${ }^{20}$ INAF Astronomical Observatory of Trieste, via Tiepolo 11, 34143 Trieste, Italy

${ }^{21}$ Graduate Institute of Astronomy, National Central University, 300 Chung-Da Rd, Chung-Li 32054, Taiwan

22 Space Science Institute, Macau University of Science and Technology, Avenida Wai Long, Taipa, Macau

${ }^{23}$ Institut für Geophysik und extraterrestrische Physik, Technische Universität Braunschweig, Mendelssohnstr. 3, 38106 Braunschweig, Germany

${ }^{24}$ Instituto de Astrofísica de Andalucía (CSIC), c/ Glorieta de la Astronomia s/n, 18008 Granada, Spain 